\title{
Detecting Denial of Severe Psychiatric Disorder: An MMPI-2 Investigation of Criminal Defendants
}

L. Thomas Kucharski

John Jay College of Criminal Justice

Paul Tang

John Jay College of Criminal Justice

Barry Rosenfeld

Fordham University

Follow this and additional works at: https://fordham.bepress.com/psych_facultypubs

Part of the Psychology Commons

\section{Recommended Citation}

Kucharski, L. Thomas; Tang, Paul; and Rosenfeld, Barry, "Detecting Denial of Severe Psychiatric Disorder: An MMPI-2 Investigation of Criminal Defendants" (2007). Psychology Faculty Publications. 18.

https://fordham.bepress.com/psych_facultypubs/18 


\title{
Detecting Denial of Severe Psychiatric Disorder: An MMPI-2 Investigation of Criminal Defendants
}

\author{
L. Thomas Kucharski, Paul Tang, and Barry Rosenfeld
}

The ability of the MMPI-2 validity scales to differentiate mentally ill defendants who denied being mentally ill from mentally ill defendants who acknowledged their illness and those without mental illness was investigated. A stepwise logistic regression analysis (LGA) was utilized to identify the most parsimonious set of predictors among the MMPI-2 validity measures. The $F$ and $K$ scales and the Es-K index significantly differentiated the denying mental illness group from those acknowledging their psychiatric difficulties with a high degree of accuracy. An ROC curve utilizing the prediction scores from the LGA yielded an area under the curve of .89. An attempt to validate the model by differentiating the denial participants from those with no mental illness resulted in a marked decline in classification accuracy. The results are discussed in terms of the utility of the MMPI-2 in the detection of under reporting of psychiatric difficulties.

Denial of psychological difficulties, personality deficits or character flaws is a common occurrence in forensic settings. Denying psychological problems in child custody determinations, civil commitment evaluations, or evaluations for probation or parole hearings are all common situations in which denial of genuine psychiatric symptoms occurs. In each of these settings, the ramifications of an incorrect assessment of the evaluatee's mental health can be substantial. Placement of a child with a mentally ill and/or dangerous parent, failing to address the treatment needs of a potentially dangerous mentally ill individual, or recommending conditional release for an offender whose mental health needs have not been recognized or met can result in life-threatening situations.

Despite the obvious motivation to deny mental disorder in many forensic settings, not all instances of denial are the result of a deliberate, conscious attempt to portray oneself as healthy for some secondary gain. Many individuals genuinely lack insight into the nature and extent of their psychological difficulties, as is often the case among individuals with somatoform disorders or personality disorders. In more extreme cases, denial of symptoms can result from the illness itself, as is common among many individuals who suffer from severe mental illnesses such as schizophrenia, mania, or delusional disorder. For example, many patients who suffer from delusional disorders become quite defensive whenever a clinician suggests that their difficulties might be the result of a mental disorder.

Research focused on the distortion of psychiatric symptoms, whether exaggeration or minimization, has largely relied on psychological tests such as the MMPI (Hathaway \& McKinley, 1983) or the MMPI2 (Butcher, Dahlstrom, Graham, Tellegen \& Kaemmer, 1989). The ability to detect symptom exaggeration with the MMPI-2 is relatively wellestablished (Berry, Baer, \& Harris, 1981; Rogers, Sewell, \& Salekin, 1994; Rogers, Sewell, Martin, \& Vitacco, 2003), but the detection of denial or minimization is less often studied. Indeed, metaanalytic studies of malingering on the MMPI-2 have typically generated much larger effect sizes than have studies of minimization (Baer, Wetter, \& Berry, 1992; Baer \& Miller, 2002). These studies have demonstrated that detecting symptom underreporting is far more difficult than detecting symptom exaggeration or malingering. This finding is not surprising, given the wide range of contexts and motivations that lead to denial of psychiatric symptoms, as well as the wide array of symptoms and disorders that might be minimized (e.g., psychotic symptoms, sexual deviance, substance abuse, impulse control problems).

L. Thomas Kucharski and Paul Tang are at John Jay College of Criminal Justice, and Barry Rosenfeld is at Fordham University. The assertions contained herein are the private views of the authors and should not be construed as official or reflecting the views of the United States Department of Justice or the Federal Bureau of Prisons. Address correspondence to L. Thomas Kucharski, Department of Psychology, John Jay College of Criminal Justice, 445 West 59 ${ }^{\text {th }}$ Street New, York, NY, 10022 (Email: tkucharski@jjay.cuny.ed). 
Furthermore, drawing conclusions from the research on underreporting is limited by the research methodologies typically employed by these studies. In a recent meta-analysis of studies of underreporting, Baer and Miller (2002) found 22 studies of underreporting involving the MMPI-2. Twentyone of these 22 studies utilized a simulation design where students or psychiatric patients were asked to either respond honestly or to attempt to "fake good" on the MMPI-2 (i.e., deny symptoms or present themselves in an unrealistically positive light). However, these simulation designs have limited external validity, since the extent to which the research findings can be generalized to actual forensic evaluation context is often unknown.

When actual forensic samples are studied, the primary method has been to study the differential prevalence of reported symptoms. For example, Walters (1988) compared federal inmates undergoing a parole evaluation with those entering group therapy comparing their MMPI profiles, assuming that those in the parole group had an incentive to deny psychopathology. Although he found significant differences between the groups, the magnitude of these group differences was small. Ultimately, differential prevalence designs leave unanswered questions about whether the subject's response pattern is due to the incentive to fake good or from the real absence of pathology or some combination of the two. Moreover, research on symptom distortion is often dramatically influenced by the base rate of the phenomena in question. More recent studies (Nicholson et al., 1997) have utilized statistical procedures that are relatively independent of the base rate (e.g., ROC analyses), but such studies have been extremely rare with regard to the detection of under reporting.

The present study attempted to address many of these limitations observed in previous research by using a known-groups design and incorporating dataanalytic procedures that are relatively independent of the base rate. Specifically, we studied three groups of forensic participants, including mentally ill individuals who acknowledged their symptoms and/ or disorder, a group of mentally ill individuals who denied psychiatric difficulties, and a third group of defendants referred for evaluation who had no serious identifiable mental disorder.

\section{METHOD}

\section{Participants}

Data were obtained from the medical records of criminal defendants referred for psychological evaluation by the Federal Courts. The basis for referral was to establish competency to stand trial, criminal responsibility, or provide aide in sentencing decision making. Each defendant was evaluated by one or more licensed clinical psychologists, all of whom had between 5 and 20 years of experience. Diagnoses were made in accordance with the Diagnostic and Statistical Manual of Mental Disorders, $4^{\text {th }}$ Edition (APA, 1994). Records were reviewed to establish a) psychiatric history and previous diagnoses and b) presenting symptoms. Cases in which a clear, documented history of severe psychiatric disorder (i.e., an Axis I disorder other than substance abuse) or, alternatively, the unequivocal absence of any such history, along with the presence of sufficient psychological test data to permit analysis, were included in these analyses.

Three groups of participants were selected from 309 consecutive referrals. Excluded were 83 defendants whose MMPI-2 results were suggestive of malingering or exaggeration $(\mathrm{F}>95)$ and those whose protocols were not interpreted due to a VRIN raw score $\geq 13, T R I N \geq T=80$ or 30 or more omitted items $(N=21)$. An additional 44 of the potential participants were excluded as they were not administered the MMPI-2 either due to refusal, language other than English or Spanish, low intellectual functioning, or the discretion of the evaluator. This left a sample of 161 total defendants. The "No Mental Illness" group consisted of 65 defendants (38 males and 27 females) who had no history of outpatient or inpatient psychiatric treatment and denied all symptoms of major mental illness. None of these individuals were diagnosed (by the evaluating clinician) with any Axis I mental disorder other than substance abuse. The "Accepting" mental illness group was comprised of 49 defendants (19 males and 30 females) with a documented history (prior to incarceration on the instant offense) of psychiatric hospitalization, who were diagnosed with a major mental disorder by the evaluating clinician, and acknowledged their mental illness during the evaluation. The third group of defendants, the 
"Denying" mental illness group, included 47 (39 males, 8 females) individuals, most of whom had a prior history of psychiatric treatment, presented psychotic symptoms during the evaluation but vehemently denied being mentally ill and refused any attempt to present a defense based on their mental condition.

\section{Procedures}

The clinical, social and legal histories of all participants were collected from clinical interviews lasting between 3 and 8 hours total, interviews with family members, review of medical records, and interviews with the prosecuting and defense attorneys, probation, pre trial services officers and police officials. Psychological testing included individual administration of the MMPI-2 under standard procedures. In addition, the Rorschach Inkblot test, intelligence assessment, and neuropsychological screening were conducted on individual cases when clinically indicated. All data collected was archival, routinely collected during the process of the forensic evaluation, and was not anticipated for use in specific research investigations. Hence, informed consent was not obtained.

The MMPI-2 standard validity indices, L (lie), $\mathrm{F}$ (infrequency), $\mathrm{K}$ (correction), Fb (back infrequency), and F-K were used as predictor variables. Other predictor variables included several newer scales such as Fp (Infrequency psychopathology, Arbisi \& Ben-Porath, 1995, 1997), S (superlative scale, Butcher \& Han, 1995) and the Es-K index (Borum \& Stock, 1993). MMPI-2 protocols with VRIN (variable response inconsistency) greater than or equal to $13, \mathrm{~F}$ scale $\mathrm{T}>95, \mathrm{~F}-\mathrm{K}$ index $>13$, TRIN $\mathrm{T}>80$ or more than 30 omitted items were excluded as these protocols were potentially invalid due to marked inconsistent responding, deliberate exaggeration, or scale deflation due to omitted items. Review of the clinical and social histories, all collateral interviews and assignment to groups were conducted blind to the MMPI-2 results.

\section{Statistical Analyses}

Initial analyses used frequency and ANOVA models to a) assess for group differences in demographic and clinical characteristics, b) identify patterns of group differences on the MMPI-2 validity indices, and c) assess group differences on the 10 MMPI-2 clinical scales. Subsequent stepwise logistic regression analysis was used to identify the most parsimonious set of predictors that best differentiated the mentally ill participants who denied the presence of a mental disorder from those who acknowledged their illness. Predictor variables were drawn from the set of MMPI-2 variables and indices that have been used to identify defensive or invalid responding (described above). The resulting model was subsequently applied to an ROC model in order to ascertain the overall predictive accuracy of this regression model at various alternative "cut-off" points. Finally, this logistic regression model was used to generate "prediction" scores for the participants without evidence of a mental disorder in order to ascertain the efficacy of this set of MMPI2 variables in differentiating psychologically intact from defensive mentally ill participants. These prediction scores were then used to create a second ROC curve corresponding to the utility of the logistic regression model.

\section{RESULTS}

\section{Sample Characteristics}

Of the 161 participants included in this analysis (96 male, 65 female), the average age at the time of evaluation was 37.0 (range: $18-72, S D=10.5$ ). Most participants were Caucasian $(N=88,54.7 \%)$, with 52 black (32.3\%), 16 Hispanic $(9.9 \%)$ and 5 from other ethnic backgrounds (3\%). The average number of years of education was 11.9 (range: 4 to 19 years, $S D=2.6$ ) with 61 participants having less than a high school education (37.9\%), 50 with a high school diploma or equivalent (31\%) and the remainder $(N$ $=50,37.9 \%$ ) having some education beyond high school. The majority of participants had some prior mental health treatment, with $62(38.5 \%)$ having one or more prior psychiatric hospitalization and 71 (44.1\%) having had prior outpatient mental health treatment (41 had both).

The three groups differed significantly in terms of gender, $\chi^{2}(2, N=161)=19.53, p=.0001$, and years of education, $F(2,158)=5.52, p=.005$, but not age, $F(2,158)=2.08, p=.13$, or race, $\chi^{2}(2, N=$ 
$158)=9.77, p=.461$ (see Table 1). Specifically, the No Mental Illness group had a significantly lower level of education than the two groups of mentally ill offenders/defendants whereas the Accepting mental illness group was more likely to be female. The two samples of mentally ill offenders/defendants (Accepting, Denying) were largely similar in terms of diagnostic classifications (see Table 1), although some of the less frequently observed diagnoses did reach statistical significance (e.g., delusional disorder was significantly more common among the Denying group).

\section{Group Differences in Individual MMPI-2 Scales}

Univariate analyses of variance (ANOVA) of subject group (Accepting, Denying, and No Mental Illness) with each of the MMPI-2 validity scales as dependent variables revealed significant group differences for each of the validity scales except the $\mathrm{L}$ and Fp scales (see Table 2). Post-hoc tests revealed that the Accepting group scored significantly higher $(p<.05)$ than both the No Mental Illness and the Denying groups on the F scale, the F-K index, and the $\mathrm{Fb}$ scale and significantly lower on the $\mathrm{S}$ scale. The Accepting group also scored significantly lower on the K scale than the Denying group, but neither group differed significantly from the No Mental Illness group. Of note, there were no significant differences between the Denying and No Mental Illness groups on any of the validity scales or indices.

An ANOVA comparison of the three groups treating the MMPI-2 clinical scales as dependent variables also revealed significant group differences on all of the clinical scales except MasculinityFemininity (Mf) and Mania (Ma). Post-hoc tests indicated that the Accepting group scored significantly higher than both the Denying and No Mental Illness groups on five of the ten clinical scales (Hypochondriasis or Hs, Hysteria or Hy, Paranoia or $\mathrm{Pa}$, Schizophrenia or $\mathrm{Sc}$ and Social Introversion or Si). In addition, all three groups differed from one another on the Depression (D) and Psychoasthenia (Pt), with the No Mental Illness group falling between the Accepting and Denying groups. This same pattern emerged on the Psychopathic Deviate (Pd) scale, although only the Accepting and Denying groups differed significantly (the No Mental Illness group did not differ from either of these two groups). Thus, of the ten clinical scales, only two differed significantly between the Denying and No Mental Illness groups, D and Pt.

\section{Multivariate Prediction of Denial}

A stepwise logistic regression analysis was used to identify the most parsimonious set of predictors among the MMPI-2 validity indices that best differentiated the Accepting Mental Illness from the Denial of Mental Illness groups. This model yielded three predictor variables, F, K and Es-K, each of which provided a significant, unique contribution to differentiating the two groups (see Table 3 ). This model was statistically significant, Wald $\chi^{2}(1, N=$ $96)=54.59, p<.001 ;\left(\right.$ Cox \& Snell $\left.R^{2}=.43\right)$, and resulted in a correct classification of $82.3 \%$ of the cases. An ROC curve utilizing the prediction scores from the logistic regression analysis for these two groups yielded an area under the curve of .89 (see Figure 1).

When this logistic regression model was used to distinguish the Denying and the No Mental Illness groups, however, the predictive accuracy deteriorated markedly. The resulting classification analysis revealed that $71 \%$ of cases were correctly classified (see Table 4), resulting in a Kappa coefficient of .44 and an area under the curve of .60. Inspection of the classification table revealed that the lower predictive accuracy was due to moderate levels of denial in our No Mental Illness comparison group and misclassification of those without mental illness as denying psychiatric difficulties.

\section{DISCUSSION}

Denial of mental disorder and/or the successful portrayal of psychological well being in individuals with serious psychological disturbance can have important consequences for the administration of justice. Deliberate, conscious efforts to fake good in civil commitment decisions may, if successful, result in the release of participants at risk for violence. In other settings, successful denial of mental disorder can result in a conviction for a mentally ill defendant who refuses to disclose the psychotic basis for his/ her criminal behavior, effectively preventing the 
Table 1

Sample Demographic and Clinical Characteristics

\begin{tabular}{lllll}
\hline & $\begin{array}{c}\text { Accepting } \\
(n=49)\end{array}$ & $\begin{array}{l}\text { Denying } \\
(n=47)\end{array}$ & $\begin{array}{c}\text { None } \\
(n=65)\end{array}$ & $p^{\text {a }}$ \\
\hline Male (\%) & $19(39 \%)$ & $39(83 \%)$ & $38(58 \%)$ & .0001 \\
Age (mean, $S D)$ & $38.8(8.9)$ & $39.4(11.0)$ & $35.4(11.1)$ & .13 \\
Years of Education (mean, $S D)$ & $12.0(2.5)$ & $12.7(2.6)$ & $11.2(2.4)$ & .005 \\
Race (\% Caucasian) & $28(57 \%)$ & $28(60 \%)$ & $32(49 \%)$ & .46 \\
Diagnosis & & & & .85 \\
$\quad$ Schizophrenia & $22(45 \%)$ & $22(47 \%)$ & $0(0 \%)$ & .0007 \\
$\quad$ Delusional disorder & $2(4 \%)$ & $14(30 \%)$ & $0(0 \%)$ & .02 \\
$\quad$ Schizoaffective disorder & $6(12 \%)$ & $0(0 \%)$ & $0(0 \%)$ & .67 \\
$\quad$ Bipolar disorder & $10(20 \%)$ & $8(17 \%)$ & $0(0 \%)$ & .03 \\
Other affective disorder & $5(10 \%)$ & $0(0 \%)$ & $0(0 \%)$ & .68 \\
Other psychotic disorder & $4(8 \%)$ & $5(11 \%)$ & $0(0 \%)$ & \\
\hline
\end{tabular}

Table 2

Group Differences in MMPI-2 Validity and Clinical Scales

\begin{tabular}{llllrl}
\hline Scale & Accepting & Denying & None & $F$ & $p^{\mathrm{a}}$ \\
\hline $\mathrm{L}$ & $60.4(12.2)$ & $62.3(12.8)$ & $60.0(13.6)$ & 0.46 & .63 \\
$\mathrm{~F}$ & $80.3(16.3)^{\mathrm{a}}$ & $61.1(15.9)^{\mathrm{b}}$ & $69.2(20.9)^{\mathrm{b}}$ & 13.56 & .0001 \\
$\mathrm{~K}$ & $44.7(14.0)^{\mathrm{a}}$ & $51.9(11.7)^{\mathrm{b}}$ & $49.1(12.3)$ & 3.93 & .022 \\
$\mathrm{Fp}$ & $68.8(19.9)^{\mathrm{a}}$ & $62.9(15.5)$ & $62.4(20.1)$ & 1.82 & .16 \\
$\mathrm{Fb}$ & $84.7(22.3)^{\mathrm{a}}$ & $61.8(17.6)^{\mathrm{b}}$ & $70.5(22.6)^{\mathrm{b}}$ & 14.42 & .0001 \\
$\mathrm{~S}$ & $43.6(8.6)^{\mathrm{a}}$ & $52.4(11.1)^{\mathrm{b}}$ & $49.4(11.1)^{\mathrm{b}}$ & 9.12 & .0001 \\
$\mathrm{~F}-\mathrm{K}$ & $2.9(13.3)^{\mathrm{a}}$ & $-8.6(9.7)^{\mathrm{b}}$ & $-4.2(11.3)^{\mathrm{b}}$ & 12.10 & .0001 \\
$\mathrm{Es}-\mathrm{K}$ & $11.5(6.9)^{\mathrm{a}}$ & $17.5(4.5)^{\mathrm{b}}$ & $16.7(7.6)^{\mathrm{b}}$ & 12.02 & .0001 \\
& & & & \\
$\mathrm{Hs}$ & $71.4(14.7)^{\mathrm{a}}$ & $57.2(11.5)^{\mathrm{b}}$ & $60.7(14.0)^{\mathrm{b}}$ & 14.59 & .0001 \\
$\mathrm{D}$ & $75.0(15.7)^{\mathrm{a}}$ & $56.0(11.7)^{\mathrm{b}}$ & $63.7(14.3)^{\mathrm{c}}$ & 22.14 & .0001 \\
$\mathrm{Hy}$ & $69.2(15.5)^{\mathrm{a}}$ & $56.7(11.7)^{\mathrm{b}}$ & $60.1(14.5)^{\mathrm{b}}$ & 10.35 & .0001 \\
$\mathrm{Pd}$ & $72.5(13.8)^{\mathrm{a}}$ & $62.8(11.4)^{\mathrm{b}}$ & $68.0(13.3)$ & 6.85 & .001 \\
$\mathrm{Mf}$ & $54.2(12.1)$ & $49.6(9.4)$ & $50.0(9.7)$ & 3.03 & .051 \\
$\mathrm{~Pa}$ & $82.4(19.6)^{\mathrm{a}}$ & $66.2(14.7)^{\mathrm{b}}$ & $71.4(20.8)^{\mathrm{b}}$ & 9.35 & .0001 \\
$\mathrm{Pt}$ & $72.8(14.3)^{\mathrm{a}}$ & $54.1(8.6)^{\mathrm{b}}$ & $62.7(15.7)^{\mathrm{c}}$ & 23.12 & .0001 \\
$\mathrm{Sc}$ & $77.6(14.5)^{\mathrm{a}}$ & $61.0(11.2)^{\mathrm{b}}$ & $66.4(18.7)^{\mathrm{b}}$ & 14.46 & .0001 \\
$\mathrm{Ma}$ & $58.7(13.5)$ & $56.6(12.6)$ & $55.6(11.9)$ & 0.87 & .42 \\
$\mathrm{Si}$ & $64.7(11.1)^{\mathrm{a}}$ & $50.7(12.1)^{\mathrm{b}}$ & $56.0(13.6)^{\mathrm{b}}$ & 15.63 & .0001 \\
\hline
\end{tabular}

Note: different subscripts indicate significant $(p<.05)$ group contrast on post-hoc tests. 
Table 3

Logistic Regression Model Distinguishing Mentally Ill Defendants who Accept their Mental Illness from those who Deny Mental Illness

\begin{tabular}{lcccccr}
\hline Variable & $\mathrm{B}$ & Wald $\chi^{2}$ & $d f$ & $p$ & Odds Ratio & \multicolumn{1}{c}{$95 \% C I$} \\
\hline $\mathrm{F}$ & -.05 & 7.15 & 1 & .008 & 0.96 & $.90-.96$ \\
$\mathrm{~K}$ & .09 & 10.68 & 1 & .0001 & 1.16 & $1.04-1.16$ \\
Es-K & .27 & 15.90 & 1 & .0001 & 1.32 & $1.15-1.49$ \\
\hline
\end{tabular}

Table 4

Classification Accuracy Based on Logistic Regression Models

Model 1: Accepting versus Denying Mental Illness

$\begin{array}{lcc} & & \text { Actual Group } \\ \text { Predicted Group } & \text { Denying } & \text { Accepting } \\ \text { Denying } & 38 & 8 \\ \text { Accepting } & 9 & 41\end{array}$

Model 2: Denying versus No Mental Illness

Predicted Group

Denying

Denying

Actual Group

Not Mental Illness

Figure 1

ROC Curve Predicting Denial of Mental Illness based on MMPI-2 Validity Scales/Indices

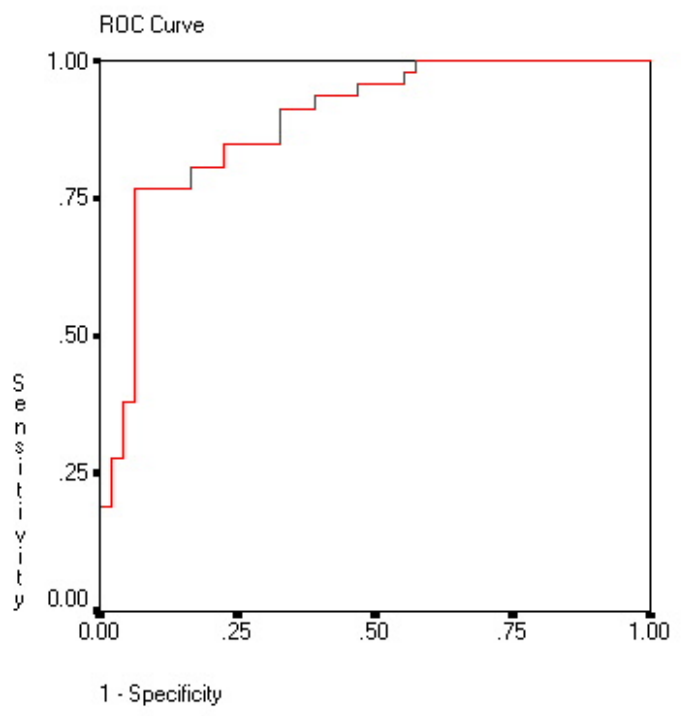


application of a psychiatric defense that is otherwise appropriate. In child custody settings, the failure to identify a parent's mental illness can result in a placement that violates the goal of the child's best interest and, in the extreme, may jeopardize the child's safety.

Although a large and ever-growing body of empirical research has focused on the ability to detect exaggerated psychiatric symptoms (i.e., malingering), far less attention has focused on the identification of symptom minimization or denial. This study utilized the MMPI-2, perhaps the most commonly-used and widely-researched psychological test, to differentiate three groups of criminal defendants: those with a history of mental illness who acknowledge psychiatric symptoms, those with a history of mental illness (and a disorder confirmed by clinical evaluation) who denied mental illness, and a third group with no known, reported, or suspected mental disorder.

The MMPI-2 performed quite well in differentiating defendants who acknowledged versus denied their mental illness. Three of the MMPI-2 validity scales/indices, F, K, and the Es-K index, accurately differentiated the mentally ill individuals who accepted versus denied mental illness with a high degree of predictive accuracy. However, when this model was used to distinguish mentally ill individuals who denied mental illness from those without a mental disorder, the predictive utility of this model dropped markedly, suggesting that the real-world utility of these variables may be substantially less than would be hoped. Further, although our reliance on ROC analyses to assess predictive utility reduces the confounding influence of base rates, base rate information is nevertheless crucial in determining the real-world utility of any predication model. For example, a review of Table 4 reveals that both models have, in these samples, roughly comparable negative predictive accuracy but the positive predictive accuracy (likelihood that a prediction of denial of mental disorder is correct) of these models differed substantially (and would deteriorate further if the base rate of denial decreased from the $42 \%$ rate in this study).

Despite the differing levels of predictive utility, these results support the existing literature on the ability of the MMPI-2 to differentiate defensive psychiatric patients from non-mentally ill individuals. However, the vast majority of these studies have relied on simulation designs rather than a known groups design, as used in the present investigation. Hence, the demonstration that the MMPI-2 validity indices can successfully differentiate known groups of mentally ill defendants who accept versus deny mental disorder represents a substantial improvement upon the existing literature. Of course, somewhat more humbling results were observed when this model was used to differentiate individuals who denied symptoms from those with no known mental disorder, as many individuals without any mental disorder were incorrectly classified as "deniers" based on these validity scales. Thus, the real-world utility of MMPI-2 validity scales/indices in distinguishing defensive versus genuinely psychologically healthy individuals is likely to be far less than the existing research literature would suggest.

Understanding the relatively poorer performance of the MMPI-2 in distinguishing mentally ill individuals who deny illness from non-mentally ill individuals is complicated by several methodological limitations in this study. A careful review of the scale means for the "no mental illness" group reveals substantial levels of reported psychopathology, despite the fact that none of these individuals had a history of psychiatric treatment and were deemed, by the evaluating clinician, to not be mentally ill. Of course, the possibility always exists that these individuals were merely superior "deniers" who were capable of masking their symptoms despite intensive clinical interviews, collateral contacts, and record review. However, such a possibility seems quite unlikely (particularly since most of the defendants studied would likely have been motivated to exaggerate, not deny their symptoms). Likewise, it is also possible that some of the "Accepting" group were in fact exaggerating or fabricating symptoms and were not genuinely mentally ill. Because no "gold standard" exists for determining the presence of genuine mental illness, any incorrect classifications would likely diminish the predictive utility of these models. Finally, because individuals who deny symptoms of a mental disorder are not likely to acknowledge their motivation for their defensiveness, it is not possible to assess whether different patterns exist among individuals who deliberately mask symptoms from those who deny symptoms for other reasons (e.g., unconscious motives or as a function of their mental disorder). 
Despite methodological limitations, this study represents one of the few attempts to apply a known groups design to the study of defensiveness in criminal defendants. The MMPI-2 demonstrated adequate ability to distinguish mentally ill individuals who accept versus deny their illness, but was somewhat less useful in distinguishing non-mentally ill individuals from those denying psychiatric symptoms. Further research using alternative assessment tools (e.g., projective versus objective psychological tests, structured clinical interviews, biological markers) may yield multifaceted models that are superior to the MMPI-2 alone. In the meantime, clinicians should exercise caution in making decisions based solely on psychological test data without careful analysis of other sources of information.

\section{REFERENCES}

American Psychiatric Association. (1994). Diagnostic and statistical manual of mental disorder $\left(4^{\text {th }} \mathrm{ed}\right.$.). Washington, D.C.: American Psychiatric Association.

Arbisi, P. A. \& Ben-Porath, Y. S. (1995). An MMPI-2 infrequency response scale for use with psychopathological populations. Psychological Assessment, 7, 424-431.

Arbisi, P. A. \& Ben-Porath, Y. S. (1997). Characteristics of the MMPI-2 $\mathrm{F}(\mathrm{p})$ scale as a function of diagnosis in an inpatient sample of veterans. Psychological Assessment, 9, 102-105.
Baer, R. A., Wetter, M. W. \& Berry, D. T. R. (1992). Detection of underreporting of psychopathology on the MMPI: A meta-analysis. Clinical Psychology Review, 12, 509-525.

Baer, R. A. \& Miller, J. (2002). Underreporting of psychopathology on the MMPI-2: A meta-analytic review. Psychological Assessment, 14, 16-26.

Berry, D. T. R., Baer, R. A. \& Harris, M. J. (1981). Detection of malingering on the MMPI: A meta-analytic review. Clinical Psychology Review, 11, 585-598.

Borum, R. \& Stock, H. V. (1993). Detection of deception in law enforcement applicants. Law and Human Behavior, 17, 157166.

Butcher, J. N., Dalstrom, W. G., Graham, J. R., Tellegen, A. M. \& Kaemmer, B. (1989). MMPI-2: Manual for administration and scoring. Minneapolis, University Press.

Butcher, J. N., \& Han, K. (1995). Development of an MMPI-2 scale to assess the presentation of self in a superlative manner: the S scale. In J. N. Butcher \& C. O. Spielberger (Eds.), Advances in personality assessment (Vol. 10, pp. 25-50). Hillside, N.J.: Erlbaum.

Nicholson, R. A., Mouton, G. J., Bagby, R. M., Buis, T., Peterson, S. A. \& Buigas, R. A. (1997). Utility of MMPI-2 indicators of response distortion receiver operating characteristic analysis. Psychological Assessment, 9, 471-479.

Rogers, R., Sewell, K. W., \& Salekin, K. T. (1994). A metaanalysis of malingering on the MMPI-2. Assessment, 1, 227-238.

Rogers, R., Sewell, K.W., Martin, M.A., \& Vitacco, M.J. (2003). Detection of feigned mental disorders: A meta-analysis of the MMPI-2 and malingering. Assessment, 10, 160-177.

Walters, G. D. (1988). Assessing dissimulation and denial on the MMPI in a sample of maximum security inmates. Journal of Personality Assessment, 52, 465-474. 